\section{Psychological Medicine}

cambridge.org/psm

\section{Original Article}

Cite this article: Sallis HM, Croft J, Havdahl A, Jones HJ, Dunn EC, Davey Smith G, Zammit S, Munafò MR (2021). Genetic liability to schizophrenia is associated with exposure to traumatic events in childhood. Psychological Medicine 51, 1814-1821. https://doi.org/ $10.1017 /$ S0033291720000537

Received: 11 September 2019

Revised: 4 February 2020

Accepted: 25 February 2020

First published online: 1 April 2020

Key words:

ALSPAC; childhood trauma; gene-environment correlation; MoBa; psychosis

Author for correspondence:

Hannah M. Sallis, E-mail: hannah.sallis@ bristol.ac.uk (c) The Author(s) 2020. Published by Cambridge University Press. This is an Open Access article, distributed under the terms of the Creative Commons Attribution licence (http:// creativecommons.org/licenses/by/4.0/), which permits unrestricted re-use, distribution, and reproduction in any medium, provided the original work is properly cited.

\section{Genetic liability to schizophrenia is associated with exposure to traumatic events in childhood}

\author{
Hannah M. Sallis ${ }^{1,2,3,4} \mathbb{D}$, Jazz $\mathrm{Croft}^{1}$, Alexandra Havdahl' ${ }^{2,5,6}$, Hannah J. Jones ${ }^{1,4}$, \\ Erin C. Dunn ${ }^{7,8,9}$, George Davey Smith ${ }^{2,4}$, Stanley Zammitt ${ }^{1,4,10}$ and Marcus

\section{R. Munafò 2,3,4}

\begin{abstract}
${ }^{1}$ Centre for Academic Mental Health, Population Health Sciences, Bristol Medical School, University of Bristol, Bristol, UK; ${ }^{2} \mathrm{MRC}$ Integrative Epidemiology Unit, University of Bristol, Bristol, UK; ${ }^{3}$ UK Centre for Tobacco and Alcohol Studies, School of Psychological Science, University of Bristol, Bristol, UK; ${ }^{4} \mathrm{NIHR}$ Biomedical Research Centre, University Hospitals Bristol NHS Foundation Trust and University of Bristol, Bristol, UK; ${ }^{5} \mathrm{Nic}$ Waals Institute, Lovisenberg Diaconal Hospital, Oslo 0853, Norway; ${ }^{6}$ Department of Mental Disorders, Norwegian Institute of Public Health, Oslo N-0213, Norway; ${ }^{7}$ Psychiatric and Neurodevelopmental Genetics Unit, Center for Genomic Medicine, Massachusetts General Hospital, Boston, MA, USA; ${ }^{8}$ Department of Psychiatry, Harvard Medical School, Boston, MA, USA; ${ }^{9}$ Broad Institute of Harvard and MIT, Cambridge, MA, USA and ${ }^{10}$ MRC Centre for Neuropsychiatric Genetics and Genomics, Cardiff University, Cardiff, UK
\end{abstract}

\title{
Introduction
}

There is a wealth of literature from observational studies showing an association between childhood trauma and psychotic illness. Recent studies have suggested that exposure to interpersonal violence or neglect in childhood can increase the risk of psychotic symptoms by twothree times (Cunningham, Hoy, \& Shannon, 2016; McGrath et al., 2017; Trotta, Murray, \& Fisher, 2015; van Dam et al., 2012; Varese et al., 2012). These findings suggest that childhood trauma is a causal environmental risk factor for both sub-clinical psychotic symptoms and psychotic disorder. However, the relationship between childhood trauma and psychosis is complex and could be explained, in part, by gene-environment correlation.

Gene-environment correlation reflects the association between an individual's genotype and their environment (Jaffee \& Price, 2008). There are three types of gene-environment correlations, commonly referred to as: passive, evocative (or reactive), and active (or selective) (Jaffee \& Price, 2008). In each case, there is an observable association between genotype and environment, although the presumed mechanisms underlying these correlations are distinct. Passive gene-environment correlation occurs when genetic information passed from parent to child is associated with the environment in which the child is raised. For example, it could be that parents with a genetic predisposition to psychosis may be more liable to behaviours that create an environment in which the child is subject to exposure to traumatic stress. In this case, as for evocative and active gene-environment correlation, trauma would be a marker for genetic risk of psychosis, not necessarily a cause of psychosis (Jaffee \& Price, 
2008). In addition to passive gene-environment correlation, parental genotype can also influence child phenotype via the genetic information that is not transmitted. This process is known as dynastic effects, where parent phenotype can influence their offspring's outcomes (Brumpton et al., 2019; Davies et al., 2019; Kong et al., 2018). Evocative gene-environment correlation is the association between an individual's genetic predisposition to a certain behaviour, and reactions of others to that behaviour. For example, the child may exhibit traits that elicit reactions of others around them - e.g. harsher parenting or victimisation by peers. Active gene-environment correlation occurs when an individual seeks out a particular environment based on their genetic information. For example, individuals with increased genetic predisposition to sensation-seeking behaviours may be more likely to seek out riskier environments, such as associating with individuals who misuse substances, and hence have increased risk of substance misuse themselves. In our psychosis example, children with certain traits may seek out environments in which they may be more vulnerable to exposure to traumatic events. The relative importance of each type of correlation is presumed to change with development, with passive most influential earlier in life and active becoming more important as individuals begin to select their own environment (Scarr \& McCartney, 1983).

Building from these insights, we hypothesised that the observed association between childhood trauma and psychosis is partly due to gene-environment correlation. In all three situations, an association between the occurrence of psychosis (due to increased genetic liability) and trauma could occur as a result of gene-environment correlation, meaning without trauma being causal. However, it is also possible that gene-environment correlation can be part of a causal chain, a fact utilised by Mendelian randomisation, which relies on such correlations to infer causality (Davey Smith \& Ebrahim, 2005; Gage et al., 2016). Whilst a number of lines of evidence support the view that traumatic experiences can have a causal effect on psychosis risk, it is important to understand the extent of gene-environment correlation in this relationship as: (i) this will lead to more accurate estimates of any causal effect of trauma on psychosis, and (ii) this can support the argument to provide support to individuals at high genetic risk to minimise the occurrence of traumatic events. To examine this possibility, we investigated genetic correlations between psychosis and trauma by testing the association between polygenic scores (PGS) for schizophrenia and childhood trauma exposure across two international longitudinal cohorts.

\section{Methods}

\section{Avon Longitudinal Study of Parents and Children (ALSPAC)}

ALSPAC is a longitudinal pregnancy cohort which aimed to recruit all pregnant women in the former county of Avon with an expected due date between April 1991 and December 1992. Detailed information has continued to be collected on mothers, partners and children in the cohort, this process has been described in detail elsewhere (Boyd et al., 2013; Fraser et al., 2013; Northstone et al., 2019). Ethics approval for the study was obtained from the ALSPAC Ethics and Law Committee and the Local Research Ethics Committees. Informed consent for the use of data collected via questionnaires and clinics was obtained from participants following the recommendations of the ALSPAC Ethics and Law Committee at the time. Please note that the study website contains details of the data that are available through a fully searchable data dictionary and variable search tool.

\section{Measures of childhood trauma}

The ALSPAC measures of childhood trauma are described in detail elsewhere (Croft et al., 2019). In brief, trauma exposure was collected prospectively from 0 to 17 years and supplemented by retrospective data collected at 22 years. Information was collected on several categories of trauma exposure and questions were selected to reflect experiences that would likely be highly upsetting to anyone encountering them. In ALSPAC, childhood trauma was derived using parent and child responses to a range of questionnaires collected across childhood and adolescence. In early childhood (before 5 years), only parent-reported data were available, while in adolescence (between 11 and 17 years) measures of trauma were mainly child-reported. When both childand parent-report were available, these were combined to derive the exposure to trauma measure.

A composite measure of 'any trauma' was derived spanning the whole of childhood and adolescence (0-17 years) alongside trauma at several intervals (0-4.9 years, 5-10.9 years and 11-17 years; Fig. 1). Trauma exposures were also separated into distinct domains: bullying, domestic violence, sexual abuse, emotional neglect, emotional cruelty and physical cruelty. A detailed description of the items included in each trauma measure can be found in the eMethods of Croft et al. (2019).

\section{Polygenic scores (PGS) for schizophrenia liability}

Genotyped data were available for 7977 children and 8196 mothers in the ALSPAC study. Data were also available on a subset of fathers within the cohort $(n=1481)$. Details of genotyping and quality control measures are available in online Supplementary Materials.

PGS for schizophrenia liability were derived for mothers, fathers and children with genetic data available and were standardised prior to analyses. Schizophrenia PGS were derived as described in Jones et al. (2018). These scores were based on publicly available summary statistics published by the Psychiatric Genomics Consortium (PGC) (34 241 cases and 45604 controls) (Ripke et al., 2014). Overlapping single-nucleotide polymorphisms (SNPs) in the genome-wide association study (GWAS) summary statistics and ALSPAC imputed genetic data were identified and linkage disequilibrium (LD) clumping was used to identify independent genetic variants, with priority given to those with a stronger association in the PGC discovery GWAS. Weighted PGS were calculated using the effect estimates from the PGC GWAS, with SNPs being included in the PGS depending on the strength of the association in the original PGC GWAS. Thirteen schizophrenia association $p$-value thresholds were used ranging from $p<0.5$ to $p<5 \times 10^{-8}$ (online Supplementary Table S1), with a greater number of SNPs included as the $p$-value thresholds became less conservative.

\section{Statistical analyses}

\section{Multiple imputation}

To maximise sample size and reduce selection bias due to attrition, multiple imputation was used to impute missing values in the trauma data. The imputation model included a broad range of variables related to trauma or variables known to be associated with sample missingness, in addition to the schizophrenia PGS 
ALSPAC

$\mathrm{MoBa}$

Fig. 1. Timeline of data collection for the trauma measures in ALSPAC and MoBa.

and all variables included in any of the analysis models. A full description of the imputation strategy can be found in the online Supplementary Materials.

\section{Analysis}

Analyses were performed in Stata 15 (StataCorp, 2017). Unadjusted logistic regression was used to investigate the strength of association between schizophrenia PGS and experiencing childhood trauma. Our main analyses focused on PGS at a threshold of $p<0.05\left(\mathrm{PGS}_{0.05}\right)$ as this explains the most variability in genetic liability for schizophrenia in other samples (Ripke et al., 2014).

Four main outcomes were examined; these were trauma measured across childhood and adolescence (0-17 years) and then at specific intervals during this period ( $0-4.9$ years, $5-10.9$ years and 11-17 years). The associations between schizophrenia $\mathrm{PGS}_{0.05}$ and specific trauma subtypes were also investigated. Each analysis was repeated using $\mathrm{PGS}_{0.05}$ derived for the mothers, fathers and children.

\section{Sensitivity analyses}

We performed three sensitivity analyses. First, we examined the association between all PGS thresholds from $p<0.5$ to $p<5 \times 10^{-8}$ and exposure to trauma measured between 0 and 4.9 years of age. By restricting our analyses to this younger age group, it was expected that attrition would be minimal, and effects observed within this subset would be less prone to selection bias. Analyses were also performed using the complete-case data, to check consistency of results with the imputed dataset.

Second, we performed unadjusted logistic regression of child $\mathrm{PGS}_{0.05}$ on trauma exposure, restricting the analysis to children with data available on mother and father genotype. We repeated this model, adjusting for mother and father genotype to estimate the association between child PGS and trauma, independent of parental genotype.

Finally, we examined whether the association with trauma exposure was specific to genetic liability for schizophrenia or if it extended to psychiatric traits more generally. We created maternal and child $\mathrm{PGS}_{0.05}$ for several additional psychiatric phenotypes, described below, and conducted unadjusted logistic regressions to investigate the association between these scores with exposure to 'any trauma' at age $0-4.9$ years. These additional phenotypes were attention-deficit hyperactivity disorder (ADHD) (Demontis et al., 2019), autism spectrum disorder (ASD) (Grove et al., 2019), bipolar disorder (Stahl et al., 2019), major depressive disorder (MDD) (Wray et al., 2018), neuroticism (Luciano et al., 2018), an updated schizophrenia risk score (Pardiñas et al., 2018)
0-17 years: Self- and parent-reported measures

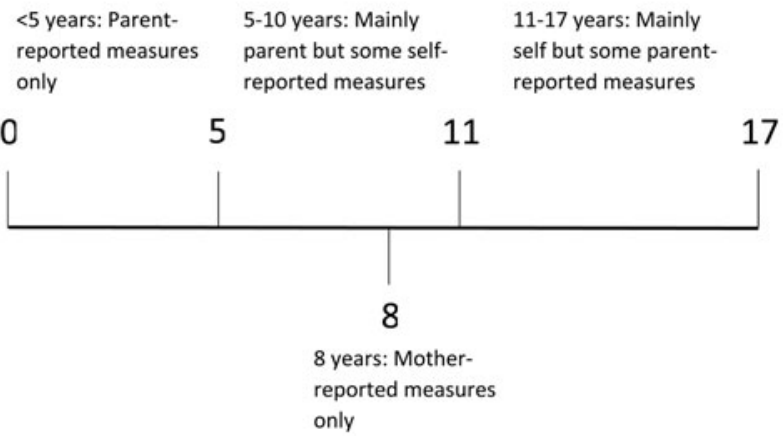

and a joint cross-disorder phenotype consisting of five major psychiatric disorders (Cross-Disorder Group of the Psychiatric Genomics Consortium et al., 2013).

\section{Replication in $\mathrm{MoBa}$}

We attempted to replicate the PGS-trauma association in an independent sample, the Norwegian Mother, Father and Child Cohort Study (MoBa). MoBa is a prospective population-based pregnancy cohort study conducted by the Norwegian Institute of Public Health (Magnus et al., 2016). Participants were recruited from all over Norway from 1999 to 2008 . In $40.6 \%$ of the pregnancies, women consented to participate. The cohort now includes 114500 children, 95200 mothers and 75200 fathers. The current analyses were approved by The Regional Committees for Medical and Health Research Ethics (reference number 2016/1702) (further details are available in online Supplementary Materials).

Details of genotyping and quality control measures in MoBa are available in online Supplementary Materials. $\mathrm{PGS}_{0.05}$ for schizophrenia liability were calculated for mothers, fathers and children with genetic data available at the time of analysis (15 208 children, 14804 mothers and 15198 fathers). Childhood trauma was measured in $\mathrm{MoBa}$ at age 8 years using maternal responses to questions selected to reflect experiences that would be highly upsetting to anyone encountering them (Fig. 1). There were genetic data and trauma data available for 7244 children. Bullying was defined as being subjected to beating, kicking or other violence by other children in the past year $(n=$ $1665,23.0 \%$ ), emotional neglect as not usually letting the child know when he/she is doing a good job at something $(n=124$, $1.7 \%)$ and physical abuse as being subjected to beating, kicking or other violence by adults in the past year $(n=49,0.7 \%)$. In addition, the three questions were used to derive a composite measure of 'any trauma' ( $n=1765,24.3 \%)$. Details of the questions and prevalence in the full $\mathrm{MoBa}$ cohort are available in online Supplementary Table S3.

Within MoBa, the association between $\mathrm{PGS}_{0.05}$ and trauma exposure was assessed in complete cases using logistic regression adjusting for chip, batch and 10 principle components.

\section{Results}

\section{Sample description}

After imputation, 13595 participants had data on childhood trauma within ALSPAC. By 17 years, $72.8 \%$ of the sample were 
Table 1. Proportion with reported trauma across each age range in ALSPAC participants with and without genetic data

\begin{tabular}{lcccc}
\hline $\begin{array}{l}\text { Any } \\
\text { childhood } \\
\text { trauma }\end{array}$ & $\begin{array}{c}\text { Whole } \\
\text { sample } \\
(N=13595)\end{array}$ & $\begin{array}{c}\text { With } \\
\text { genetic } \\
\text { data }^{\mathrm{a}} \\
(n=9946)\end{array}$ & $\begin{array}{c}\text { Without } \\
\text { genetic } \\
\text { data } \\
(n=3649)\end{array}$ & $p$-value \\
\hline 0-4.9 years & 29.2 & 27.2 & 34.5 & $<0.001$ \\
\hline 5-10.9 years & 50.1 & 47.9 & 56.2 & $<0.001$ \\
\hline $11-17$ years & 47.9 & 46.1 & 52.7 & $<0.001$ \\
\hline $0-17$ years & 72.8 & 70.8 & 78.5 & $<0.001$ \\
\hline
\end{tabular}

${ }^{\mathrm{a}}$ This refers to individuals with either child or maternal genetic data available.

reported to have experienced any trauma. The levels of trauma were lower in those with genetic data $(n=9946)$, with $70.8 \%$ of participants experiencing trauma compared to $78.5 \%$ of those without genetic data $(n=3649)$ (Table 1$)$. The prevalence of each trauma domain ranged from $9.8 \%$ (emotional neglect) to $35.3 \%$ (bullying), and from $27.2 \%$ (age $0-4.9$ years) to $70.8 \%$ (0-17 years) for 'any trauma' (online Supplementary Table S2).

In $\mathrm{MoBa}, 42236$ participants had information on trauma measured at 8 years; of these individuals, $25.3 \%$ reported experiencing any trauma, with 'bullying' being the most frequently endorsed exposure (online Supplementary Table S3).

\section{Association between schizophrenia PGS and experiencing trauma across childhood and adolescence}

Within ALSPAC and across PGS scores derived based on child and mother data, we found evidence of an association between the schizophrenia $\mathrm{PGS}_{0.05}$ and increased exposure to childhood trauma at ages 0-4.9 years, 5-10.9 years and 11-17 years, as well as across childhood and adolescence [0-17 years: odds ratio $\left(\mathrm{OR}_{\mathrm{Child}}\right) 1.14,95 \%$ confidence interval $(\mathrm{CI})$ 1.08-1.20, $p=8.4 \times 10^{-6} ; \mathrm{OR}_{\text {Mother }} 1.13,95 \%$ CI $1.06-1.20, p=8.5 \times 10^{-5}$; Table 2]. When using the smaller sample of fathers with schizophrenia $\mathrm{PGS}_{0.05}$ available, the effect estimates were compatible with those observed using the mother and child $\mathrm{PGS}_{0.05}$, although there was no strong evidence of an association (0-17 years: $\mathrm{OR}_{\text {Father }} 1.04,95 \%$ CI $0.92-1.17, p=0.549$; Table 2).

\section{Subtypes of trauma}

When investigating the association between schizophrenia $\mathrm{PGS}_{0.05}$ and different types of trauma within ALSPAC, we found both maternal and child $\mathrm{PGS}_{0.05}$ were associated with most trauma subtypes (Table 3). However, we found no robust evidence of an association between either maternal or child $\mathrm{PGS}_{0.05}$ and bullying. We also observed a similar pattern of effects when using paternal $\mathrm{PGS}_{0.05}$ (Table 3).

\section{Sensitivity analyses}

When using both parental and child PGS generated using SNPs associated with schizophrenia at a range of $p$-value thresholds, we observed a positive association between increased PGS and experiencing trauma at all $p$-value thresholds (online Supplementary Table S1).
Analyses were repeated using complete case data. The rates of trauma were comparable across each domain, and particularly at the youngest age group ( $0-4.9$ years) where attrition was lowest (online Supplementary Table S2). Similarly, when looking at the association between $\mathrm{PGS}_{0.05}$ and trauma across the life course using complete-case data, effect estimates were largest at the youngest ages where attrition was minimal (online Supplementary Table S4), and these were in a consistent direction, although slightly attenuated, compared to the effects estimated when using the imputed data. The pattern of effects remained consistent at each age group, with evidence of an association between the schizophrenia $\mathrm{PGS}_{0.05}$ and increased exposure to childhood trauma (online Supplementary Table S5).

In the restricted sample of individuals with data on both mother and father genotype, we found strong evidence of an association between increased child $\mathrm{PGS}_{0.05}$ and trauma, with the observed effect sizes larger than those in the analyses containing the full child sample (Table 4). After adjusting for both maternal and paternal genotype, the strength of evidence of association was reduced, although effect estimates were consistent with those estimated in the full child sample (Table 4).

We also examined the association of alternative psychiatric $\mathrm{PGS}_{0.05}$ with childhood trauma. We found strong evidence of an association between the MDD, neuroticism, cross-disorder phenotype and the updated schizophrenia PGS and childhood trauma, with similar effect sizes found for most of these associations (online Supplementary Table S6). For both cross-disorder and $\mathrm{MDD}$, these associations were stronger when using the child $\mathrm{PGS}_{0.05}$ than the maternal $\mathrm{PGS}_{0.05}$. There was also some evidence of an association between childhood trauma and ADHD and bipolar disorder $\mathrm{PGS}_{0.05}$, but this was weaker. Evidence for these findings was consistent when using either maternal or child $\mathrm{PGS}_{0.05}$.

\section{Replication in an independent sample}

Within MoBa, there was some evidence of association between the schizophrenia $\mathrm{PGS}_{0.05}$ and trauma exposures at age 8 years (Table 5). The effect estimates for any trauma were largely consistent with those estimated in ALSPAC at a similar age (5-10.9 years; Table 2).

\section{Discussion}

The main finding of this study is that PGS for schizophrenia liability were associated with increased exposure to childhood trauma within two independent and international birth cohorts. This finding was consistent when using both parental and child PGS, suggesting that it is unlikely to be solely driven by direct genetic effect from either parents or children. We also found that genetic liability to a range of additional psychiatric traits was associated with a greater trauma exposure. This highlights that individuals at higher genetic risk for poor mental health may benefit from supports that decrease their risk of trauma. It may also suggest that the original GWASs of these psychiatric traits are not only identifying variants associated with the trait of interest, but are also reflecting the effects of risk factors for these traits.

As GWASs become larger and thus statistically more powerful, they can identify variants of increasingly small effect that are robustly associated with the disorder being studied. For example, prior to 2014, GWAS had identified around 30 loci associated with schizophrenia (Ripke et al., 2014). With the publication of 
Table 2. Unadjusted association between schizophrenia $\mathrm{PGS}_{0.05}$ and exposure to any trauma in ALSPAC

\begin{tabular}{|c|c|c|c|c|c|c|c|c|c|}
\hline \multirow[b]{2}{*}{ Age range (years) } & \multicolumn{3}{|c|}{ Child PGS $(N=7426)$} & \multicolumn{3}{|c|}{ Mother PGS $(N=7380)$} & \multicolumn{3}{|c|}{ Father PGS $(N=1215)$} \\
\hline & OR & $95 \% \mathrm{Cl}$ & $p$-value & OR & $95 \% \mathrm{Cl}$ & $p$-value & OR & $95 \% \mathrm{Cl}$ & $p$-value \\
\hline $5-10.9$ & 1.07 & $1.01-1.13$ & 0.018 & 1.10 & $1.03-1.16$ & 0.002 & 1.06 & $0.95-1.20$ & 0.291 \\
\hline $11-17$ & 1.13 & $1.07-1.20$ & $4.4 \times 10^{-5}$ & 1.15 & $1.08-1.22$ & $1.3 \times 10^{-5}$ & 0.99 & $0.87-1.11$ & 0.808 \\
\hline
\end{tabular}

$\mathrm{OR}$, odds ratio; $\mathrm{PGS}$, polygenic score; $\mathrm{Cl}$, confidence interval.

Table 3. Unadjusted association between schizophrenia $\mathrm{PGS}_{0.05}$ and subtypes of trauma across childhood and adolescence (age 0-17 years) in ALSPAC

\begin{tabular}{|c|c|c|c|c|c|c|c|c|c|}
\hline \multirow[b]{2}{*}{ Type of trauma } & \multicolumn{3}{|c|}{ Child PGS $(N=7426)$} & \multicolumn{3}{|c|}{ Mother PGS $(N=7380)$} & \multicolumn{3}{|c|}{ Father PGS $(N=1215)$} \\
\hline & OR & $95 \% \mathrm{Cl}$ & $p$-value & OR & $95 \% \mathrm{Cl}$ & $p$-value & OR & $95 \% \mathrm{Cl}$ & $p$-value \\
\hline Domestic violence & 1.07 & $1.01-1.13$ & 0.028 & 1.16 & $1.09-1.23$ & $3.4 \times 10^{-6}$ & 1.07 & $0.92-1.25$ & 0.385 \\
\hline Sexual abuse & 1.15 & $1.03-1.29$ & 0.012 & 1.12 & $1.00-1.25$ & 0.043 & 1.11 & $0.89-1.38$ & 0.343 \\
\hline Emotional cruelty & 1.16 & $1.09-1.24$ & $3.49 \times 10^{-6}$ & 1.15 & $1.08-1.24$ & $6.7 \times 10^{-5}$ & 1.05 & $0.90-1.22$ & 0.534 \\
\hline Physical cruelty & 1.12 & $1.05-1.20$ & $7.9 \times 10^{-4}$ & 1.16 & $1.08-1.24$ & $1.7 \times 10^{-5}$ & 1.08 & $0.93-1.25$ & 0.298 \\
\hline
\end{tabular}

$\mathrm{OR}$, odds ratio; $\mathrm{PGS}$, polygenic score; $\mathrm{Cl}$, confidence interval.

Table 4. Association between child PGS 0.05 and trauma at each time point - analysis restricted to subset with maternal and paternal PGS

\begin{tabular}{|c|c|c|c|c|c|c|}
\hline \multirow[b]{2}{*}{ Age range (years) } & \multicolumn{3}{|c|}{ Child PGS unadjusted $(N=1526)$} & \multicolumn{3}{|c|}{ 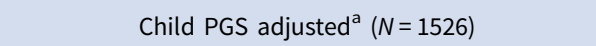 } \\
\hline & OR & $95 \% \mathrm{Cl}$ & $p$-value & OR & $95 \% \mathrm{Cl}$ & $p$-value \\
\hline $5-10.9$ & 1.10 & $0.98-1.22$ & 0.094 & 1.05 & $0.92-1.21$ & 0.474 \\
\hline $11-17$ & 1.15 & $1.03-1.29$ & 0.015 & 1.14 & $0.99-1.31$ & 0.069 \\
\hline
\end{tabular}

OR, odds ratio; PGS, polygenic score; $\mathrm{Cl}$, confidence interval.

adjusted for maternal and paternal $\mathrm{PGS}_{0.05}$.

a large meta-analysis performed by the Schizophrenia Working Group of the PGC (Ripke et al., 2014) this increased to 108 loci in 2014 and to 145 loci in 2018 (Pardiñas et al., 2018). However, with these gains in power comes the potential to detect small effects that are not direct genetic effects, but rather may reflect the effects of modifiable risk factors for the disorder under investigation (Gage et al., 2016). For example, a GWAS of lung cancer by Amos and colleagues identified a variant located in the nicotinic receptor gene cluster CHRNA5-A3-B4 (Amos et al., 2008). This locus is known to be robustly associated with smoking quantity, a well-known risk factor for lung cancer (Tobacco \& Genetics Consortium, 2010; Ware, van den Bree, \& Munafò, 2011). It therefore seems possible that if exposure to childhood trauma is a risk factor for schizophrenia, then variants associated with trauma exposure could be picked up in the original schizophrenia GWAS. In addition to acting as an instrument for schizophrenia, this PGS could therefore be acting as a proxy for factors that could contribute to trauma exposure, and the association we observe between the PGS for schizophrenia liability and childhood trauma could occur as a result. If sufficient data and robust instruments were to become available, approaches such as Mendelian randomisation for mediation could be used to contribute to these questions (Relton \& Davey Smith, 2012).

In addition to investigating the association with a single composite measure of trauma, we also examined several subdomains of trauma to further disentangle the relationship. These subdomains were: bullying, domestic violence, sexual abuse, emotional neglect, emotional cruelty and physical cruelty. When using both maternal and child PGS, we observed strong evidence of an association between increased schizophrenia PGS and all domains of childhood trauma across the life course, with the exception of bullying. Paternal PGS again generally showed a consistent direction of effect but, due to the smaller sample size, analyses were underpowered to detect effects. With the exception of bullying, each of our trauma subdomains is likely to occur within the home environment. Bullying, which often takes place at school 
Table 5. Association between $\mathrm{PGS}_{0.05}$ and exposure to trauma in the Norwegian MoBa

\begin{tabular}{|c|c|c|c|c|c|c|c|c|c|}
\hline \multirow[b]{2}{*}{ Type of trauma } & \multicolumn{3}{|c|}{ Child PGS $(N=7244)$} & \multicolumn{3}{|c|}{ Mother PGS $(N=7009)$} & \multicolumn{3}{|c|}{ Father PGS $(N=7153)$} \\
\hline & OR & $95 \% \mathrm{Cl}$ & $p$-value & OR & $95 \% \mathrm{Cl}$ & $p$-value & OR & $95 \% \mathrm{Cl}$ & $p$-value \\
\hline Bullying & 1.09 & $1.03-1.15$ & 0.003 & 1.09 & $1.03-1.15$ & 0.003 & 1.07 & $1.01-1.13$ & 0.019 \\
\hline Emotional neglect & 0.87 & $0.73-1.04$ & 0.122 & 1.12 & $0.93-1.34$ & 0.221 & 0.84 & $0.70-1.00$ & 0.046 \\
\hline Physical abuse & 1.33 & $1.00-1.76$ & 0.047 & 0.96 & $0.72-1.28$ & 0.785 & 1.61 & $1.21-2.13$ & 0.001 \\
\hline Any trauma & 1.08 & $1.02-1.14$ & 0.005 & 1.10 & $1.04-1.16$ & 0.001 & 1.06 & $1.00-1.11$ & 0.052 \\
\hline
\end{tabular}

OR, odds ratio; PGS, polygenic score; $\mathrm{Cl}$, confidence interval.

and in the neighbourhood, showed little evidence of an effect in the ALSPAC cohort. Within ALSPAC, the bullying domain predominantly captured peer bullying, with only a single item on sibling bullying. In contrast, we did observe some association within MoBa, however, the bullying measure in MoBa includes exposure to violence from siblings. This suggests that there may be something specific to the home environment that is influencing this PGS-trauma exposure association, indicative of gene-environment correlation. If evocative gene-environment correlation was at play, we may expect to observe an association with bullying in addition to the other types of trauma. Equally, if active gene-environment correlation was occurring, we may expect to see a stronger association with the child PGS compared to the parental PGS. Given that we do not see either, and instead find that the association appears specific to more likely home-based exposures, it is possible that some form of passive gene-environment correlation is occurring, and that trauma could be a marker of genetic liability for later psychosis as well as a causal risk factor for psychosis. If the association that we observe in the home environment is due to parental traits, then establishing which of these traits play a role in shaping the greater 'traumagenic' environment could enable us to target modifiable factors to reduce their occurrence and impact. Although sufficient data are not currently available, analyses investigating between-sibling effects could go some way to disentangling these associations.

After adjusting analyses for parental PGS to investigate the effect of child genotype independently of parental genetic effects, we found that at younger ages the child genotype effect estimates attenuated, while during adolescence effect estimates remained consistent. Since trauma is predominantly parent-reported at younger ages with an increasing number of self-reported measures in adolescence, one explanation is that this could be capturing reporting bias. An alternative argument is that child genotype becomes more influential for later trauma as children age; hence, a transition occurs from a passive gene-environment correlation to a more active gene-environment correlation model. Previous findings suggest that those exposed to trauma in early life are at greater risk of revictimisation in adolescence, which may also contribute to this observation (Fisher et al., 2015). If true, prevention measures should focus on parents and supporting a healthy homelife earlier on in childhood and targeting the children themselves as they approach adolescence. Developing prevention measures to reduce vulnerability to trauma among individuals with a higher genetic liability to poor mental health, if timed right, could help to reduce subsequent onset of mental illness among these high-risk individuals.

We also investigated the association between childhood trauma with child and maternal PGS for a number of alternative psychiatric phenotypes, using the latest available data (Pardiñas et al., 2018). We observed an association between several scores and childhood trauma; however, the strongest effects were with PGS for schizophrenia and neuroticism. Results from the updated schizophrenia GWAS were comparable with the main analysis using PGS from the 2014 GWAS. There was strong evidence when looking at child PGS for MDD and a cross-disorder phenotype, but these effects were smaller when using maternal PGS. It seems likely that the association with childhood trauma is not necessarily specific to schizophrenia, but genetic predisposition to poor mental health in general. These findings are in line with recent work by Schoeler et al. (2019) and Leppert et al. (2019) who both found an association with genetic vulnerability to mental illness and exposure to bullying and other stressful life events.

The observed association between genetic liability for poor mental health and exposure to childhood trauma does not suggest that the majority of individuals with a mental health disorder will expose their children to traumatic events. The measure of trauma used in our study included a range of different types of trauma exposure and were experienced by a large proportion of participants, including those with a low genetic liability. The mechanisms through which the association between genetic liability and childhood trauma acts could be such that individuals with a higher genetic liability for poor mental health outcomes may end up in more deprived neighbourhoods where exposure to trauma may be more common (Solmi, Lewis, Zammit, \& Kirkbride, 2019), or that they engage in relationships where domestic violence is more likely to occur. These mechanisms would again suggest a form of passive gene-environment correlation, with the association arising between the genetic information passed from parent to child, and the environment in which the child is raised. If this is the case, this suggests that those with higher genetic liability for mental illness may be most vulnerable to these effects and should be offered increased support to decrease their risk of trauma.

Another potential implication of our findings is that the observational association between childhood trauma and subsequent psychotic events may be partly due to genetic confounding, meaning that any causal effect of trauma on psychosis is somewhat weaker than the observed association between childhood trauma and subsequent psychosis would indicate. In a previous study using the ALSPAC cohort, we showed that the association between trauma and psychotic experiences was not attenuated by adjusting for either child or mother genetic risk for schizophrenia (Croft et al., 2019). Therefore, whilst the results from this present study indicate that exposure to trauma is higher where parents have a higher genetic risk for psychiatric disorders, this 
does little to challenge the theory that trauma has a causal effect on psychosis risk. Whilst measurement error in the PGS could have led to residual confounding in the study by Croft et al., and led to an overestimate of association, it seems unlikely that gene-environment correlation offers a plausible explanation for the trauma-psychosis relationship described in observational studies to date.

There are several limitations in our study that should be considered. First, there was substantial missing data in the trauma variable which could have resulted in selection bias. However, we used multiple imputation to impute our sample and maximise sample size, and the effect estimates in the complete cases at the youngest age bracket were comparable with those from the imputed dataset. In ALSPAC, attrition has been shown to be patterned by genetic risk factors (Taylor et al., 2018), therefore given the increased possibility of selection bias when using data at the older ages where attrition was greater, we also restricted this analysis to trauma measured up to age 5 years. We observed similar results at this age to the overall effect estimates, with a positive association between increased PGS and experiencing trauma. Second, the sub-set of fathers with genetic data was limited and so these father-based analyses were underpowered. We aimed to improve this by replicating in MoBa. Third, the trauma variables in the MoBa cohort were measured at age 8 years only and not as extensively as in the ALSPAC cohort. Therefore, we were unable to recreate completely comparable subdomains of trauma to disentangle the gene-environment correlation in this cohort. For example, in MoBa the question used to measure bullying ('Has your child been subjected to beating, kicking or other violence by other children in the past 12 months?') has the potential to capture both victims of bullying and the bullies themselves, if their victims were to retaliate. However, previous research has suggested that most bullies are also victims of bullying and therefore would likely be captured within this definition regardless (Lereya, Copeland, Zammit, \& Wolke, 2015). Fourth, in MoBa and the early childhood measures of trauma in ALSPAC were parent-reported only. It would be interesting to repeat these analyses comparing the parental PGS with parent-reported trauma exposure and child PGS with self-reported trauma exposure. However, in ALSPAC at the ages where self-reported measures were available (age 8 years onwards), the direction of effect remained consistent. Including analysis of parent's own reported exposure to childhood trauma may also provide a more complete context for understanding the interplay between genetic risk and environmental factors that contribute to the risk of traumatic exposure during childhood.

\section{Conclusion}

Analyses across two international birth cohorts indicate that genetic liability for schizophrenia, as well as other psychiatric phenotypes, is associated with childhood trauma. This could suggest that GWAS for these psychiatric traits are not only identifying variants associated with these traits, but may also reflect risk factors associated with them. We also found evidence to suggest the association between genetic liability for schizophrenia and exposure to trauma has some specificity to the home environment and suggests that youth at higher genetic risk might require greater resources/support to ensure they grow up in a healthy environment.

Supplementary material. The supplementary material for this article can be found at https://doi.org/10.1017/S0033291720000537.
Acknowledgements. ALSPAC: We are extremely grateful to all the families who took part in this study, the midwives for their help in recruiting them, and the whole ALSPAC team, which includes interviewers, computer and laboratory technicians, clerical workers, research scientists, volunteers, managers, receptionists and nurses.

$\mathrm{MoBa}$ : We thank the Principle Investigators of the major genotyping projects, Per Minor Magnus (NIPH) and Pål Njølstad (University of Bergen) for providing genotype data for the replication in MoBa. MoBa is supported by the Norwegian Ministry of Health and Care Services and the Ministry of Education and Research. We are grateful to all the participating families in Norway who take part in this on-going cohort study. We thank the Norwegian Institute of Public Health (NIPH) for generating genomic data in MoBa as part of the HARVEST collaboration, supported by the Research Council of Norway (NRC) (\#229624). We also thank the NORMENT Centre for providing genotype data, funded by NRC (\#223273), South East Norway Health Authority and KG Jebsen Stiftelsen. Further we thank the Center for Diabetes Research, the University of Bergen for providing genotype data and performing quality control and imputation of the data funded by the ERC AdG project SELECTionPREDISPOSED, Stiftelsen Kristian Gerhard Jebsen, Trond Mohn Foundation, NRC, the Novo Nordisk Foundation, the University of Bergen, and the Western Norway health Authorities (Helse Vest). We thank Laurie Hannigan (Lovisenberg Diaconal Hospital) and Elizabeth Corfield (NIPH) for assisting with the QC and data preparation.

Financial support. This work was supported by the Medical Research Council and the University of Bristol (MC_UU_00011/1 and MC_UU_00011/7) and by the National Institute for Health Research (NIHR) Biomedical Research Centre at the University Hospitals Bristol National Health Service Foundation Trust and the University of Bristol. A comprehensive list of grants funding is available on the ALSPAC website (http://www.bristol.ac.uk/alspac/external/documents/grant-acknowledgements. pdf). GWAS data were generated by Sample Logistics and Genotyping Facilities at Wellcome Sanger Institute and LabCorp (Laboratory Corporation of America) using support from 23andMe. The views expressed in this publication are those of the authors and not necessarily those of the National Health Service, the National Institute for Health Research or the Department of Health.

MRM and HMS are members of the UK Centre for Tobacco and Alcohol Studies, a UKCRC Public Health Research: Centre of Excellence. The UK Medical Research Council and Wellcome (Grant ref: 102215/2/13/2) and the University of Bristol provide core support for ALSPAC. This publication is the work of the authors and they will serve as guarantors for the contents of this paper.

MoBa is supported by the Norwegian Ministry of Health and Care Services and the Ministry of Education and Research. AH was supported by the South-Eastern Norway Regional Health Authority (2018059 and 2020022).

This work was also conducted with support from the National Institutes of Health Award R01MH113930 (Dunn).

Conflict of interest. None.

\section{References}

Amos, C. I., Wu, X., Broderick, P., Gorlov, I. P., Gu, J., Eisen, T., ... Houlston, R. S. (2008). Genome-wide association scan of tag SNPs identifies a susceptibility locus for lung cancer at 15q25.1. Nature Genetics, 40(5), 616-622. https://doi.org/10.1038/ng.109.

Boyd, A., Golding, J., Macleod, J., Lawlor, D. A., Fraser, A., Henderson, J., ... Davey Smith, G. (2013). Cohort profile: The 'children of the 90s' - the index offspring of the Avon Longitudinal Study of Parents and Children. International Journal of Epidemiology, 42(1), 111-127. https://doi.org/10. 1093/ije/dys064.

Brumpton, B., Sanderson, E., Hartwig, F. P., Harrison, S., Vie, G. Å., Cho, Y., ... Davies, N. M. (2019). Within-family studies for Mendelian randomization: Avoiding dynastic, assortative mating, and population stratification biases. BioRxiv. https://doi.org/10.1101/602516.

Croft, J., Heron, J., Teufel, C., Cannon, M., Wolke, D., Thompson, A., ... Zammit, S. (2019). Association of trauma type, age of exposure, and frequency in childhood and adolescence with psychotic experiences in early 
adulthood. JAMA Psychiatry, 76(1), 79. https://doi.org/10.1001/jamapsychiatry.2018.3155.

Cunningham, T., Hoy, K., \& Shannon, C. (2016). Does childhood bullying lead to the development of psychotic symptoms? A meta-analysis and review of prospective studies. Psychosis, 8(1), 48-59. https://doi.org/10.1080/ 17522439.2015.1053969.

Davey Smith, G., \& Ebrahim, S. (2005). What can Mendelian randomisation tell us about modifiable behavioural and environmental exposures? BMJ, 330(7499), 1076-1079. https://doi.org/10.1136/bmj.330.7499.1076.

Davies, N. M., Howe, L. J., Brumpton, B., Havdahl, A., Evans, D. M., \& Davey Smith, G. (2019). Within family Mendelian randomization studies. Human Molecular Genetics, 28, R170-R179. https://doi.org/10.1093/hmg/ddz204.

Demontis, D., Walters, R. K., Martin, J., Mattheisen, M., Als, T. D., Agerbo, E., ... Neale, B. M. (2019). Discovery of the first genome-wide significant risk loci for attention deficit/hyperactivity disorder. Nature Genetics, 51(1), 6375. https://doi.org/10.1038/s41588-018-0269-7.

Fisher, H. L., Caspi, A., Moffitt, T. E., Wertz, J., Gray, R., Newbury, J., ... Arseneault, L. (2015). Measuring adolescents' exposure to victimization: The environmental risk (E-risk) longitudinal twin study. Development and Psychopathology, 27, 1399-1416. https://doi.org/10.1017/s0954579415000838.

Fraser, A., Macdonald-Wallis, C., Tilling, K., Boyd, A., Golding, J., Davey Smith, G., ... Lawlor, D. A. (2013). Cohort profile: The Avon Longitudinal Study of Parents and Children: ALSPAC mothers cohort. International Journal of Epidemiology, 42(1), 97-110. https://doi.org/10. 1093/ije/dys066.

Gage, S. H., Davey Smith, G., Ware, J. J., Flint, J., Munafò, M. R., \& Koifman, R. (2016). G=E: What GWAS can tell us about the environment. PLoS Genetics, 12(2), e1005765. https://doi.org/10.1371/journal.pgen.1005765.

Grove, J., Ripke, S., Als, T. D., Mattheisen, M., Walters, R. K., Won, H., ... Børglum, A. D. (2019). Identification of common genetic risk variants for autism spectrum disorder. Nature Genetics, 51(3), 431-444. https://oi. org/10.1038/s41588-019-0344-8.

Jaffee, S. R., \& Price, T. S. (2008). Genotype-environment correlations: Implications for determining the relationship between environmental exposures and psychiatric illness. Psychiatry, 7(12), 496-499. https://doi.org/10. 1016/j.mppsy.2008.10.002.

Jones, H. J., Heron, J., Hammerton, G., Stochl, J., Jones, P. B., Cannon, M., ... Zammit, S. (2018). Investigating the genetic architecture of general and specific psychopathology in adolescence. Translational Psychiatry, 8(1), 145. https://doi.org/10.1038/s41398-018-0204-9.

Kong, A., Thorleifsson, G., Frigge, M. L., Vilhjalmsson, B. J., Young, A. I., Thorgeirsson, T. E., ... Stefansson, K. (2018). The nature of nurture: Effects of parental genotypes. Science (New York, N.Y.), 359(6374), 424428. https://doi.org/10.1126/science.aan6877.

Cross-Disorder Group of the Psychiatric Genomics Consortium, Lee, S. H., Ripke, S., Neale, B. M., Faraone, S. V., Purcell, S. M., ... Wray, N. R. (2013). Genetic relationship between five psychiatric disorders estimated from genome-wide SNPs. Nature Genetics, 45(9), 984-994. https://doi.org/ 10.1038/ng.2711.

Leppert, B., Havdahl, A., Riglin, L., Jones, H. J., Zheng, J., Davey Smith, G., ... Stergiakouli, E. (2019). Association of maternal neurodevelopmental risk alleles with early-life exposures. JAMA Psychiatry, 76(8), 834-842. https:// doi.org/10.1001/jamapsychiatry.2019.0774.

Lereya, S. T., Copeland, W. E., Zammit, S., \& Wolke, D. (2015). Bully/victims: A longitudinal, population-based cohort study of their mental health. European Child and Adolescent Psychiatry, 24(12), 1461-1471. https://doi. org/10.1007/s00787-015-0705-5.

Luciano, M., Hagenaars, S. P., Davies, G., Hill, W. D., Clarke, T.-K., Shirali, M., ... Deary, I. J. (2018). Association analysis in over 329,000 individuals identifies 116 independent variants influencing neuroticism. Nature Genetics, 50 (1), 6-11. https://doi.org/10.1038/s41588-017-0013-8.

Magnus, P., Birke, C., Vejrup, K., Haugan, A., Alsaker, E., Daltveit, A. K., ... Stoltenberg, C. (2016). Cohort profile update: The Norwegian Mother and Child Cohort Study (MoBa). International Journal of Epidemiology, 45(2), 382-388. https://doi.org/10.1093/ije/dyw029

McGrath, J. J., Saha, S., Lim, C. C. W., Aguilar-Gaxiola, S., Alonso, J., Andrade, L. H., ... Wojtyniak, B. (2017). Trauma and psychotic experiences:
Transnational data from the World Mental Health survey. British Journal of Psychiatry, 211(6), 373-380. https://doi.org/10.1192/bjp.bp.117.205955.

Northstone, K., Lewcock, M., Groom, A., Boyd, A., Macleod, J., Timpson, N., \& Wells, N. (2019). The Avon Longitudinal Study of Parents and Children (ALSPAC): An update on the enrolled sample of index children in 2019. Wellcome Open Research, 4, 51. https://doi.org/10.12688/wellcomeopenres. 15132.1.

Pardiñas, A. F., Holmans, P., Pocklington, A. J., Escott-Price, V., Ripke, S., Carrera, N., ... Walters, J. T. R. (2018). Common schizophrenia alleles are enriched in mutation-intolerant genes and in regions under strong background selection. Nature Genetics, 50(3), 381-389. https://doi.org/10.1038/ s41588-018-0059-2.

Relton, C. L., \& Davey Smith, G. (2012). Two-step epigenetic Mendelian randomization: A strategy for establishing the causal role of epigenetic processes in pathways to disease. International Journal of Epidemiology, 41 (1), 161-176. https://doi.org/10.1093/ije/dyr233.

Ripke, S., Neale, B. M., Corvin, A., Walters, J. T. R., Farh, K. H., Holmans, P. A., ... O’Donovan, M. C. (2014). Biological insights from 108 schizophrenia-associated genetic loci. Nature, 511(7510), 421-427. https:// doi.org/10.1038/nature13595.

Scarr, S., \& McCartney, K. (1983). How people make their own environments: A theory of genotype greater than environment effects. Child Development, 54(2), 424. https://doi.org/10.2307/1129703.

Schoeler, T., Choi, S. W., Dudbridge, F., Baldwin, J., Duncan, L., Cecil, C. M., ... Pingault, J.-B. (2019). Multi-polygenic score approach to identifying individual vulnerabilities associated with the risk of exposure to bullying. JAMA Psychiatry, 76(7), 730-738. https://doi.org/10.1001/jamapsychiatry. 2019.0310

Solmi, F., Lewis, G., Zammit, S., \& Kirkbride, J. B. (2019). Neighborhood characteristics at birth and positive and negative psychotic symptoms in adolescence: Findings from the ALSPAC birth cohort. Schizophrenia Bulletin. https://doi.org/10.1093/schbul/sbz049.

Stahl, E. A., Breen, G., Forstner, A. J., McQuillin, A., Ripke, S., Trubetskoy, V., ... Sklar, P. (2019). Genome-wide association study identifies 30 loci associated with bipolar disorder. Nature Genetics, 51(5), 793-803. https://doi. org/10.1038/s41588-019-0397-8.

StataCorp (2017). Stata statistical software: Release 15. College Station, TX: StataCorp LLC.

Taylor, A. E., Jones, H. J., Sallis, H., Euesden, J., Stergiakouli, E., Davies, N. M., ... Tilling, K. (2018). Exploring the association of genetic factors with participation in the Avon Longitudinal Study of Parents and Children. International Journal of Epidemiology, 47(4), 1207-1216. https://doi.org/ 10.1093/ije/dyy060.

Tobacco and Genetics Consortium (2010). Genome-wide meta-analyses identify multiple loci associated with smoking behavior. Nature Genetics, 42(5), 411-417. https://doi.org/10.1038/ng.571.

Trotta, A., Murray, R. M., \& Fisher, H. L. (2015). The impact of childhood adversity on the persistence of psychotic symptoms: A systematic review and meta-analysis. Psychological Medicine, 45(12), 2481-2498. https://doi. org/10.1017/S0033291715000574.

van Dam, D. S., van der Ven, E., Velthorst, E., Selten, J. P., Morgan, C., \& de Haan, L. (2012). Childhood bullying and the association with psychosis in non-clinical and clinical samples: A review and meta-analysis. Psychological Medicine, 42(12), 2463-2474. https://doi.org/10.1017/S0033291712000360.

Varese, F., Smeets, F., Drukker, M., Lieverse, R., Lataster, T., Viechtbauer, W., ... Bentall, R. P. (2012). Childhood adversities increase the risk of psychosis: A meta-analysis of patient-control, prospective- and cross-sectional cohort studies. Schizophrenia Bulletin, 38(4), 661-671. https://doi.org/10.1093/schbul/sbs050.

Ware, J. J., van den Bree, M. B. M., \& Munafò, M. R. (2011). Association of the CHRNA5-A3-B4 gene cluster with heaviness of smoking: A meta-analysis. Nicotine \& Tobacco Research, 13(12), 1167-1175. https://doi.org/10.1093/ ntr/ntr118.

Wray, N. R., Ripke, S., Mattheisen, M., Trzaskowski, M., Byrne, E. M., Abdellaoui, A., ... Sullivan, P. F. (2018). Genome-wide association analyses identify 44 risk variants and refine the genetic architecture of major depression. Nature Genetics, 50(5), 668-681. https://doi.org/10.1038/s41588-0180090-3. 